\title{
An exploratory study of gender and male teachers in Early Childhood Education and Care (ECEC) centres in China
}

Yuwei $\mathrm{Xu}{ }^{1 *}$; Manjula Waniganayake ${ }^{2}$

${ }^{1}$ School of Education and Childhood Studies, University of Portsmouth, Portsmouth, UK

${ }^{2}$ Institute of Early Childhood, Macquarie University, Sydney, Australia

* Corresponding author. Email: yuwei.xu@port.ac.uk

Tel: +44-7794437929

Address: Room 2.06, St George’s Building, 141 High Street, Portsmouth PO1 2HY, United Kingdom

Co-author. Email: manjula.waniganayake@mq.edu.au

Tel: +61298509825

Address: Institute of Early Childhood, Level 2, Building X5B, Room 278

Balaclava Drive, Macquarie University, NSW 2109, Australia 


\section{An exploratory study of gender and male teachers in Early Childhood Education and \\ Care (ECEC) centres in China}

This paper explores the impact of gender on the employment of men in early childhood education and care (ECEC) centres through the voices of male and female Early Childhood Teachers (ECTs) working in China. Gender imbalance in the ECEC workforce is a global phenomenon, and little has been researched about it in countries such as China. This study was based on online interviews with 16 ECTs from three major cities in China. Findings indicated that ECTs' perceptions conformed largely with traditional gender stereotypes in Chinese culture. Western expectations that men's participation in ECEC could challenge traditional gender stereotypes and promote gender diversity were not reflected in either Chinese academic literature reviewed or the participants' views captured in this research. The appreciation of masculinity in Chinese culture and implied disadvantages are considered within a global context of empowering women and girls.

Key words: China; early childhood education and care; gender; male early childhood teachers; masculinity

Word count: 8,500 words

\section{Introduction}

Gender imbalance in the Early Childhood Education and Care (ECEC) workforce is a global phenomenon, with women outnumbering men in ECEC centres in most Western countries 
including Canada, Germany, New Zealand, and the United Kingdom (OECD 2014). Available research shows that the percentage of men who work in ECEC centres largely hovered around $1-3 \%$ in most European countries, with a few exceptions - such as Norway, Denmark, and Turkey that exceeded the average with a rating of over 5\% (Peeters, Rohrmann, and Emilsen 2015). These trends are also found in countries beyond Europe. For example, in the USA, 3.2\% of staff who worked in pre-schools and kindergartens were men (MENTEACH 2015). In New Zealand, the proportion of men working in ECEC has only recently increased to $2.2 \%$ (Koch and Farquhar 2015). The lack of men in ECEC is also evident in non-western countries such as China where men comprised only $2 \%$ of full-time preschool teachers (Ministry of Education of the People's Republic of China 2014). This pattern however does not reflect China's population in 2014 comprising 51.23\% men (National Bureau of Statistics of China 2015).

The scarcity of men in ECEC has been receiving increasing academic attention globally as reflected in the growth of publications published in English. This includes for example, the recent Special Issue on Gender Balance in the ECEC Workforce published by European Early Childhood Education Research Journal in 2015. A quick summary of existing literature in English consists of three main aspects. First, with the disappointing progress of government initiatives to encourage men's participation in ECEC in Western societies, researchers are reexamining why men are still scarce in this sector (Peeters 2013; Peeters, Rohrmann, and Emilsen 2015). Second, Rohrmann and Brody (2015) reviewed the methodologies used in research on men in ECEC, noting methodological variability including contradictory findings about men's contributions to ECEC. Three, scholarly literature about the importance of including men in ECEC has continued to flourish, and the inclusion of a particular focus on 
cultural discourses is applauded (Brody 2014; Brownhill 2015; Peeters, Rohrmann, and Emilsen 2015; Rohrmann and Emilsen 2015).

This article, however, is not intended as a comprehensive review of literature on gender and men's participation in ECEC. As published articles about men working in ECEC in China are rarely written in English, it is difficult to compare cultural specifics of the Chinese context with other parts of the world. This paper is therefore aimed at informing non-Chinese scholars about the impact of gender based values in the employment of male teachers in ECEC in China, and where appropriate, comparing the issues cross-culturally. Considering the lack of related empirical studies in Chinese literature, this article also contributes insights based on the findings from an exploratory study conducted with male and female Chinese Early Childhood Teachers (ECTs), as a way of enriching cultural understandings about men's participation in ECEC centres in China.

\section{Men's participation in ECEC in China}

In recent years, men's participation in ECEC in China has become a hot topic aligned with the increasing importance of and interests in ECEC in Chinese society. Since 2010, China has placed the development of ECEC as a national priority (Li, Deng, and Liu 2015), and calling on men to work in ECEC centres is seen as a way of improving the quality of ECEC. Because there is a national concern that contemporary Chinese boys are becoming increasingly 'feminized' and lacking in traditional masculine characteristics such as being brave, responsible and sporty as expected of Chinese men, the society believes that including men in the education and care of boys at this crucial period of child development during early 
childhood will help 'rebuild' Chinese men's masculinities (Cao and Wu 2016). Although no national programs or policies targeting male ECTs have been launched so far, some regional policies reflect the call for increasing employment of male ECTs within this context of 'masculinity admiration' in China. For example, in Jiangsu and Fujian Provinces, men enjoy free tertiary education by selecting to study ECEC at university/college, but this privilege is not afforded to women (Jiangsu Education 2014; MENTEACH 2015). Opponents of these policies argue that it is unfair to girls when boys enjoy 'special privileges' by working in ECEC. However, the intentions of the Chinese society to include men in ECEC centres for the benefits of socialisation of boys by men, and simultaneously raising the status of ECEC, remain unchallenged.

In recognizing this powerful discourse of 'masculinity admiration', Chinese academic literature overwhelmingly agrees that more men should be encouraged into ECEC due to their different roles and styles from female teachers' (Zhao 2016). Such differences include for instance, that male ECTs can teach children about being brave, decisive, and independent; that men think in more abstract ways, like taking risks, and are better at science and sports; and that men tend to give children more freedom in preschool activities (Li 2015; Xu 2016). In addition, because of the privileging of male ECTs as deemed by Chinese society, and simultaneously also acknowledging that men are still reluctant to choose ECEC as a career or are unable to remain employed in this sector for many years, Chinese academics have placed considerable research focus on exploring why few men choose to work in ECEC, the issues of pre-service teacher training for male ECTs, job adaptations and career developments of male ECTs, and how to retain men in ECEC (see for example, Han 2016; Li 2015; Shen 2016; Zhao 2016). 


\section{Why few men choose to work in ECEC centres in China}

Reflecting the globally recognized historical construction of ECEC as a 'feminized' job reflecting caring and mothering (Craig 2004; King 2004; Peeters 2007; Rentzou and Ziganitidou 2009; Roberts-Holmes and Brownhill 2012; Smith 2004), employment in ECEC centres in China has also long been regarded as a women's occupation (Chen 2015; Han 2016). Despite changing perceptions that men are also needed in the sector, the ECEC profession in China comprises largely women. There is still a social stigma in Chinese society that men who work with young children are 'abnormal' (Han 2016), making a career as an ECT an unattractive option for men. More importantly, Chinese society still sees men as the main breadwinners of families (Chen 2015), and the low wages paid to ECTs is a significant reason preventing men from seeking a career in the ECEC sector ( $\mathrm{Li}$ et al. 2016). Because of these two reasons, few men choose ECEC as their major when enrolling in university studies and subsequently, even less consider ECEC as their career option.

\section{Pre-service teacher training for male ECTs}

Even if some boys do end up in an ECEC major either due to personal interests, social expectations, or other incidental/individual circumstances ( $\mathrm{Li}$ et al. 2016), it has been noticed that some of these pre-service trainees would not work in ECEC centres after graduation (Chen 2015; Jing 2016). Chinese scholars blame that existing early childhood teacher education programs are not suitable for men (Chen 2015; Jing 2016). On the one hand, Chen (2015) believes that some mandatory modules such as dancing, music, and painting are difficult for 
most men attributed to their genetic characteristics. On the other hand, Jing (2016) warned that the female-dominated training environments are highly likely to be 'feminized' with a majority of female students and staff, and thereby being unsuitable for the male trainees. A genderspecific teacher education program for male ECTs is thus suggested by both Chen (2015) and Jing (2016) exclusively for enrolling men. In this way, they can produce graduates with predefined masculine characteristics, expected of male ECTs who can make full use of their 'gender advantages'.

\section{Job adaptations and career developments of in-service male ECTs}

Similar to pre-service male trainees, graduate ECTs are also deemed by Chinese researchers to encounter gendered problems in employment contexts. Many consider that men are less meticulous and patient with young children, and should be allocated to do more educational tasks and fewer of the caring tasks (Liu 2013; Shen 2016; Yang 2014). Worrying about male ECTs adopting the same 'feminized' teaching styles as their female colleagues do, Yang (2014) and Jing (2016) suggest that men should pay particular attention to a 'male' style of teaching which typically means being risky, energetic, tough; as well as specializing in areas like sports and science. Most importantly, in recognizing that male ECTs tend to quit their job after a short period of service (Li and Lu 2013; Zheng 2013), it is emphasized that institutions where those men work should provide more opportunities for male ECTs and where possible, raise their salaries (Jing 2016; Liu 2013; Shen 2016). To illustrate, Jing (2016) mentioned that male ECTs could be given more opportunities for promotion, which can then lead to both a higher social status and better pay rates for them. Liu (2013), Shen (2016) and Zhao (2015) also proposed 
organising activities and clubs specifically targeted at male ECTs to provide them with networking with other men and more tailored working environments.

To summarize, Chinese academic literature on men's participation in ECEC has a strong emphasis on essential gender differences between men and women based on traditional male stereotypes, and there is an expectation of male ECTs to work differently and contribute differently in terms of their teaching styles and roles. According to the literature analysed for this paper, it is clear that Chinese ECEC professionals want male ECTs to role model traditional masculine characteristics, so that they can teach boys to become men just like them.

Nevertheless, this expectation has always been approached by researchers from macro social perspectives, not necessarily reflecting male ECTs' interests at micro or individual levels. Neither has research considered female ECTs' opinions as key stakeholders working side-byside with these male colleagues. Additionally, among the few studies that used questionnaire surveys, case studies, or interviews with male in-service and/or pre-service ECTs to learn about their individual perceptions and experiences (Huang 2013; Li 2015; Li et al. 2016; Shen 2016), a universal understanding of gender by these researchers has confirmed traditional gender differences between men and women, and the conclusions are similar in reinforcing the gendered nature of the ECEC profession in China today. The catchphrase that men's involvement in ECEC could challenge gender stereotypes and promote gender diversity as appreciated by academics in Western societies (Connell and Pearse 2015; Peeters, Rohrmann, and Emilsen 2015; Rohrmann and Brody 2015), is rarely discussed in Chinese studies.

Taking into account these research gaps, this study interviewed both male and female ECTs to understand their perceptions on how gender influenced men's participation in ECEC in 
China. Through reflections on their gender and professional subjectivities, this research was aimed at finding out whether or not male and female ECTs challenged the Chinese gender discourses as reflected in men's participation in ECEC centres in this country.

\section{Research design}

Our study was theoretically informed by poststructuralist views that suggest gender is socially constructed through the power of social order and that individuals' subjective positioning within a social context is embodied as fluid social experiences (Blaise 2005; Connell and Pearse 2015). By adopting these perspectives, the social and cultural insights about men's participation in ECEC summarized from Chinese literature were used to situate male and female ECTs' understandings of gender when working in the ECEC sector, and to explore the possibilities of Chinese ECTs challenging gender stereotypes in their work with young children.

In this article, we set out to explore the findings from our study in addressing two primary questions about the employment of male ECTs in China:

(1) What are the perceptions of male and female ECTs in China about men's participation in ECEC centres?

(2) To what extent do these ECTs challenge traditional Chinese gender discourses when employed in ECEC centres in this country?

\section{Methods}

A qualitative research method of semi-structured interviewing was used, and 16 male and female ECTs who worked in public and private kindergartens or early learning centres based 
in Beijing, Tianjin, and Taiyuan Cities participated in our study. These three are economically among the most well developed cities in China. Public and private kindergartens, and private early learning centres are the main types of ECEC centres found in China. Public kindergartens are funded by provincial governments, and once a teacher gets a job in a public kindergarten, it usually constitutes a life-long appointment. Unlike the kindergartens where children stay for a whole day on weekdays, early learning centres offer specialized classes where children attend for only a few hours every week with their parents. The participants were equally distributed in terms of gender, region, and the nature of ECEC centres where they worked (see Table 1). [Table 1 near here]

Due to the geographical location of the participants, a popular Chinese online communication tool called 'QQ' was used to conduct the interviews on a 1:1 basis. 'QQ' enabled direct conversations with the participants online, guided by six broad questions prepared and sent to the participants in advance and extending on these based on the participants' responses during the interview. This method was deemed to be similar to face-toface interviewing and appropriate in capturing rich data based on participants' perspectives. Indeed, participants provided in-depth responses during the interviews, reflecting observations of James and Busher (2006) when they conducted email interviews with their participants. They explained that this may be because web-based interviews enable participants to be more relaxed without the formalities of being physically present with the researcher (ibid).

A brief survey questionnaire with closed questions about the participants' demographic characteristics was also sent electronically to each participant before the interview to obtain some background information consistently (see Table 1). Also included in the questionnaire 
were three open questions about the participants' attitudes towards men's participation in ECEC. Answers to these survey questions were then used to prepare for the interviews.

The participants were identified through snowball sampling (Lewis-Beck, Bryman, and Futing 2004) using the researcher's social networks. One man who previously worked as a kindergarten teacher but was now a primary school teacher, and another man who just graduated from university and was on his first employment placement in a kindergarten, were included in this research by way of capturing diverse experiences in the sector. Informed consent was confirmed orally at the point of first contact when the study was explained to each participant and signed consent was gained together when the completed questionnaire was returned to the researchers. Information about the participants was protected through the safe storage of data in password protected files and use of codes to identify each participant in the data analysis. Confidentiality of participants was also assured throughout this study and in the use of the findings in presentations and publications emerging from the study.

\section{Data analysis}

The online questionnaire data and interview transcripts were completed in Mandarin, and were then translated into English. Although acknowledging that cross-language translation may have some impacts on the analysis and presentation of the data collected, the translator's (one of the authors) familiarity with the research topic and Chinese culture, as well as his own experience as a native Chinese ECT, minimized these limitations (Twinn 1997).

A hybrid approach of inductive and deductive coding and theme development was used to identify key themes. The thematic analysis (Fereday and Muir-Cochrane 2006) yielded several 
themes in collating responses to the research questions identified previously. Themes were also identified as representative based on the frequency of their appearance within the data. When coding the data, quotations were identified as representative from each second-order theme to provide illustrative examples of the views of male and female ECTs. The range of topics arising from this analysis, presented a vibrant picture of how selected ECTs understand gender and ECEC in China today.

\section{Findings}

Findings from this research are based on the perspectives of both male and female ECTs and are presented in four sections. The first section, identifies participants' professional values and beliefs about choosing a career as an ECT. The second section consists of findings about the participants' professional knowledge, skills and personal qualities. In third section, findings reflecting the participants' perceptions about the workplace responsibilities of ECTs are presented. Finally, reflections on how other stakeholders perceived the employment of male ECTs as seen by these participants, are identified. Overall, the findings of this study illustrate the gendered nature of perceptions held by male and female ECTs, and how these Chinese ECTs constructed their gender and professional subjectivities in accordance with prevailing gender norms about men in China today.

\section{Professional values and beliefs about choosing a career as an ECT}

Participants in this research provided a mix of reasons for choosing to work as an ECT, such as their suitability, curiosity, and intention to get a steady job as a tenured ECT in a public 
kindergarten in China. The two reasons most frequently mentioned by almost all participants were their love of children/education and that they had studied ECEC as their major at university, suggesting that both male and female ECTs valued the emotional significance of their job, and the importance of fostering a sense of professional identity during pre-service training. In particular, three out of eight men referred to their gender as an advantage in getting a job as an ECT, and this in itself was a powerful motivator in choosing ECEC as their career. Although numbers were far from sufficient to generalize, participants' responses were indicative of a recent tendency that Chinese men were increasingly likely to work in ECEC. This tendency is attributed to growing competitiveness in the contemporary Chinese job market and emerging societal support recognizing the value of men's involvement in young children's education (Li et al. 2016).

Having entered the ECEC profession, the majority of the ECTs were willing to continue working in ECEC centres and expressed their ambitions in moving to managerial or research positions, such as being an ECT trainer or a centre director. Gender divisions between male and female ECTs' career prospects were not evident, implying that the prescribed expectation by Chinese society that men should be taking up senior positions in ECEC might be detrimental to female ECTs' professional progressions. The only male ECT who indicated that he was planning to leave the profession, made a rather strong statement: "I will never be a nursery teacher again. Perhaps a primary or a secondary school teacher, which is relatively easier." His words reflected a general concern about the massive workloads of ECEC teachers and the corresponding low wages they were being paid compared to their school based peers - which is a frequently mentioned deterrent to men's participation in ECEC centres in China (Chen 
2015; Li et al. 2016).

\section{Professional knowledge, skills and personal qualities}

The gender advantages of being a man that some male ECTs mentioned as a motivation to work in ECEC centres, were further reported by them to be related to their professional knowledge and skills. Whilst both male and female participants mentioned relevant knowledge about children and ECEC, as well as skills such as sports, singing, playing instruments, drawing, and dancing as their strengths, five out of eight men described their gender was an advantage in being a male role model or being popular among parents. By contrast, six out of eight female ECTs regarded their kindness and patience as advantages, with only one male ECT describing himself as being kind and patient like the female ECTs. Such gendered perceptions were even stronger when male and female ECTs described their respective personal qualities. Being meticulous, patient, gentle, rigorous, and kind were more often attached to female teachers; and male ECTs were more likely to be rough, physical, 'playful', lively and/or passionate. Two representative narratives identified from a male and a female ECT illustrate this as follows:

Female teachers are meticulous, patient and kind. But even male teachers in good temper may sometimes lose their temper when interacting with children. [...] Men are born to play, hence my dream is to be a 'child king', and play with children. Through playing, I can improve my relationship with children, and make them grow up happily. (male ECT)

Male teachers are more passionate, more able to control activities, more playful when organising daily life, and more capable of motivating children. While female teachers 
are more patient and meticulous. (female ECT)

Acknowledging those gender differences in personalities, one female teacher from Beijing said it was easier to work with male teachers because women were more judgmental. One male teacher from Taiyuan City, being the only male in his workplace, felt there seems to be a distance between him and his female colleagues, making him feel uncomfortable and lonely. There were little or no signs that having male ECTs would challenge prescribed gender characteristics of men and women in China, but rather, both male and female ECTs were found to be reinforcing gender stereotypes in this research.

\section{Workplace responsibilities of ECTs}

Gender stereotypical views held by male and female ECTs also influenced men's performance of workplace responsibilities in ECEC centres in China. Two male teachers, both working in early learning centres, each described a type of gender division of labour at work as reflected in the following comments:

We normally have two female teachers working together, or one male teacher with one female teacher. Some kids may need breast feeding during classes. Hence there should be at least one female teacher there for convenience.

Male teachers would actively undertake manual tasks to help female teachers.

Additionally, some other teachers ( 2 men and 3 women) indicated that there were some tasks that men should not be doing, particularly when working with children from birth to two years old. One female teacher, as the director of a public kindergarten in Tianjin City, described some 
roles that were more suitable for men, and provided an extensive explanation:

They[Men] are more suitable for teaching older children (4-6 years old), participating in school management and research, and taking their advantages in areas such as bilingual education, physical education, and arts education, areas that require creativity and motivate strong will. When teaching younger children, there involves more caring work which male teachers are too weak to perform and lack experience. But in order to cultivate children's qualities like hardworking and tolerance, male teachers' masculinities are needed to be modelled from, to encourage children to be like men. Men and women are essentially different, and it is necessary to take advantage of each group's merits in the workplace to bring up children.

These findings suggest that gendered stratifications were still persistent within the ECEC workforce even if men worked in this non-traditional occupation (Williams 1989).

Paradoxically, however, participants also emphasized that male and female ECTs were recruited to undertake the same responsibilities. In early learning centres, they implemented children's programs covering a variety of subjects including sports and music. In kindergartens, ECTs were responsible for both teaching and caring work - including tasks such as taking children to the toilet, although it has to be taken into account that all men who worked in kindergartens in this research were working with children above 3 (see Table 1). This paradox on the one hand indicated that gendered differences between male and female ECTs were occasionally overridden by the professional nature of the ECEC sector. On the other hand, it seems that the gender-neutral construction of ECEC work was reflected in the educational tasks than when performing caring tasks. Both the professionalization of ECEC and the distinction between education and care, reflected the gendered nature of ECEC as a profession (Warin 
2014) in China.

Worst of all, even if men and women were both allocated teaching responsibilities, all ECTs in this research agreed that there were differences between male and female ECTs in terms of their approaches to teaching. One of the most remarkable differences indicated by five male and four female participants was connected with their teaching styles: the male teachers adopted a more flexible and open style whilst female teachers employed a more reserved and disciplined approach, as illustrated by the following comments:

Female teachers may be quite reserved and stick to the synopsis. (male ECT)

Male ECTs are more daring to let children take risks and try new things. They are open-minded.

(female ECT)

Teaching methods of female teachers are exhaustive, attentively careful and more traditional.

(female ECT)

While male teachers normally use flexible teaching methods, generous but appropriate.

(female ECT)

Participants also described how male and female ECTs may teach differently as explained by one female teacher who said that: "Female teachers often give children roles like being a rabbit and a cat, while male teachers usually asked them to play soldiers, cops, guardians, etc." In contrast, a male teacher offered a different explanation by referring to disciplinary standards and the importance of male teachers teaching boys: 
Female teachers normally behave and discipline children quite a lot. As a result, some boys are gradually becoming too shy. Whilst male teachers teach kids more about perseverance and being hard-working. Boys may also be brighter when taught by men.

Another female teacher reinforced differences between male and female teachers by referring to the gendered nature of human physical attributes: "There are some physical things that women teachers cannot do, including lifting children high. Women teachers are more intimate with children, with fewer big movements."

Additionally, one female teacher stated: "Male teachers are themselves boys who would play more with the children." This perspective was mirrored by two male teachers, with one declaring, "most female teachers regard themselves as conductors in the teaching process, while males are more likely to perform themselves as children's peers." Likewise, another male ECT pointed out: "I treat kids as equals, and not regarding myself as a teacher. To children, I am an elder brother who plays games with them."

According to the examples reported above, male and female ECTs recognized the socially constructed nature of gender differences between men and women in Chinese society, and more specifically the variability between what was expected of male and female ECTs. These gender differences impacted upon ECTs' gender positioning of self, and were found to be guiding how male and female ECTs understood men's distinctive contributions to ECEC in China. For instance, all eight male teachers were positive about the differences that men can make to ECEC and to children's development, such as providing boys with male role models. As some male participants explained, more male teachers in ECEC does not indicate that men were more suitable for this work compared to women, but it does recognise particular influences that men 
could have on ECEC. Such influences are reflected in both child development and gender balance as reflected in the following interview excerpt:

As a male ECT, I would like each kindergarten to have male ECTs so that children could learn to be strong from male teachers and boys could learn to be men. [...] I see lots boys who are attached to their mothers and are less masculine. I want boys to be responsible and men since childhood.

These comments match prevailing values of what Chinese society expected from male ECTs, and confirmed the appreciation of masculinity at individual and micro levels. Except for one male teacher who already quit his job as an ECT, the other male ECTs said that they would recommend teaching in ECEC to other men. They also thought that there will be more men working in ECEC in the future.

Like their male colleagues, the eight female participants in this research were overwhelmingly supportive of men's participation in ECEC. They recognized the distinctive influences that men could have on children, and thought it was good to have men to balance the gender imbalance in the ECEC workforce in China: "Male participation could balance the gender ratio in ECEC, moderate the environments; it could also help with the construction of children's masculinities, enrich teaching styles."

However, when asked whether or not they would recommend other men to teach in ECEC, most of the female ECTs were critical, saying that this job was not suitable for all men but was only appropriate for those who loved children. By making this statement, Chinese female ECTs were challenging prevailing views of gender that regarded all men to be the same, in demonstrating masculine characteristics as described previously. 


\section{Reflections on the perspectives of other stakeholders}

In this section, findings about the participants' perspectives about how they expressed their beliefs about the way male and female ECTs were treated by the children, their parents and the community, are presented.

\section{Reflections on children's treatment of ECTs}

Seven male ECTs, together with four female ECTs, found that children treated teachers according to their gender differently. Another male and two female ECTs suggested that teachers' gender was not salient in the eyes of children below 3 years age, whilst older children may start to recognize gender differences. Two female ECTs, absolutely denied the possibility of children treating teachers differently because of their gender. For those who did recognize differences, some male and female ECTs explained that male teachers were more popular and attractive to children merely because of their gender and by being the minority at the centre. Other participants, however, attributed the differences to their teaching styles as reflected in the following comments:

In physical activities, children may take risks with male teachers' encouragement; but female teachers may have more restrictions for the sake of safety. Therefore, when children are participating in activities organised by male teachers, they are more creative; in activities organised by female teachers, they often show conformance. (male ECT)

Some naughty children are quite open-minded and fast thinking. They usually challenge teachers' patience. Female teachers correspondingly, coax children or blame them. While male 
teachers could just use their eye expressions or behaviours, letting children know that male teachers are not the ones to be bullied. (female ECT)

Additionally, two male teachers and one female teacher thought that boys were more disciplined when facing male ECTs; meanwhile another two female teachers said that some girls may be afraid of male teachers because of their physical shape or looks. Notwithstanding the strongly gendered understandings reported by most participants, these reflections on whether or not children treated teachers of different gender differently suggest that gender was not the only factor influencing teachers' constructions of their professional subjectivities.

\section{Reflections on parents' perceptions of ECTS}

Reflections about parents' expectations showed that all ECTs but one either agreed or completely agreed that parents have different expectations of male teachers. More than half the participants, revealed that parents valued male teachers' influences on child development and often preferred male teachers for their children regardless of whether they were sons or daughters. Further, male ECTs were considered less rigorous than female colleagues who were more considerate and tolerant as reflected in the following comments:

Some parents (usually mothers) may request attending male teachers' classes because mothers are more considerate towards male teachers. Mothers would blame less on male teachers than on female teachers if they have made the same mistakes. (female ECT)

Paradoxically, parents with a particular preference for male teachers were also perceived as concerned about male teachers' inabilities to care for children. As one female director explained:

Parents now have new understandings about male ECTs, and admit their certain advantages. 
However, they also have concerns about their children's care in kindergartens when taught by male ECTs - affected by traditional opinions [that men are not capable of caring].

Considering that Chinese parents have a strong influence on their children's early learning, their powerful but gendered expectations of male and female ECTs may also be crucial in affecting ECTs' construction of professional subjectivities. However, considering that parents' opinions as presented in this paper merely reflected the views of the ECTs themselves, further research is necessary to capture the perspectives of how parents felt about employing male ECTs directly.

\section{Reflections on public perceptions of ECTS}

Unlike the reflections on parents' views, the public perceptions of men working as ECTs in China demonstrated in this research were mixed. Four female participants thought that male ECTs had a higher social status and their impacts were valued. The majority of male teachers ( 5 out of 8 ) considered their social status to be moderate. The remaining 3 men and 4 women, in contrast, claimed that male ECTs had a low or poor status. These reflections indicate that individual ECTs may relate themselves to the wider social contexts in different ways.

At the same time, further explorations of this theme suggested that some social perceptions had a powerful influence on male ECTs' experiences in ECEC. For example, there were concerns about male ECTs being regarded as abnormal, 'girly' or feminized. Due to some child molestation cases reported by the media, male ECTs were also suspected of being dangerous to children. More importantly, traditional Chinese social perceptions that ECEC is primarily child care and is thus regarded as women's work, were also frequently cited by male and female 
ECTs as detriments to men's participation in the sector:

Affected by traditional views, people currently have misunderstandings about ECTs. They think ECTs are 'aunts' who care for children, and that even those who only attend secondary school are competent. If a girl works in a kindergarten after graduation from university, most people would be surprised, even feel sorry for you, doubt your competence and abilities. Not to mention boys. What is more, male ECTs are usually described as unambitious, unpromising and incapable. (female ECT)

A parent would very much like his son to be taught by a male teacher in kindergartens, but when the son grows up and is choosing his own career, the parent would never allow him to be an ECT because he/she thinks it's shameful, it's unpromising. (male ECT)

Having already chosen to work in ECEC, the male ECTs in this research might have demonstrated some resistances to these kinds of societal perceptions. Nevertheless, considering that most of these men were only at an early stage of their career, and that their professional identities may be reshaped over time, it is not possible to predict how resistant they will be in the future.

\section{Discussion}

This paper explored the professional values and beliefs, knowledge, skills, personal qualities and workplace responsibilities of both male and female ECTs in China. Their perceptions reflected a gendered positioning of the professional subjectivities of ECTs in this country. Findings indicated that some male ECTs took advantage of the gender stereotypical views of 
being a man, reinforcing traditional Chinese social expectations that men were best placed to teach boys about 'being a man' (Cao and Wu 2016). This research also shows that some male ECTs challenged gender stereotypes of Chinese society by prioritizing their love of children as a key motivator in seeking employment in the ECEC sector.

Participants' perceptions about gender differences in terms of teaching and caring, reflected their awareness and understanding of alternative perspectives on working in ECEC centres. For instance, men were believed to be less judgmental than women, that men were more openminded and women were more reserved, and that men were less meticulous, patient, rigorous than women, but were more physical, rough, and 'playful'. These differences reflected traditional views about men and women in Chinese society and mirrored to a certain extent, some of the arguments about gender differences amongst ECTs employed in Western societies (Xu 2012).

These characteristics also reflect the 'Westernization' of values in Chinese ECEC centres (Huo, Neuman, and Nanakida 2015). For instance, the Chinese ECTs' admiration of risky play within ECEC centres today, may have been influenced by research conducted in Western countries such as Norway (e.g. Sandseter 2012) and Australia (e.g. Little 2015). Nonetheless, although gender differences continue to be challenged and reshaped in the West (see for example, Brandes et al. 2015; King 2004; Robb 2001; Sak, Sak, and Yerlikaya 2015), based on the participants in this research, it appears less optimistic that in reality ECTs of both genders would challenge the established gender stereotypes in China.

Gender conformity found amongst the Chinese ECTs in this study also reflected how work responsibilities were allocated in ECEC centres. Although male and female ECTs performed 
similar tasks, some inconsistencies are noteworthy. Male ECTs were not expected to look after children under two years age, under the social construction of caring as 'women's work' in Chinese culture (Hou and Zhang 2009). There was also a tendency that the pedagogical aspects of ECEC were addressed with older children in Chinese kindergartens ( $\mathrm{Hu} 2015)$, and in these centres there was little or no separation of responsibilities between male and female ECTs. This division of care and education in ECEC is believed by Western scholars to be demonstrating artificial and narrow perspectives on teaching and learning tasks, and there is a risk of failure to provide children with coherent and comprehensive ECEC programs (Warin 2014). Our findings confirm the view that even if men were working in ECEC centres, for now, it remains a gendered workforce in China. How to challenge the entrenched gendered distinctions between care and education in Chinese ECEC centres, requires further investigation.

Within this context, it is understandable that when asked to comment about male participation in ECEC, participants offered strongly gendered answers, both positively and negatively. These perceptions reflected paradoxical views about men's involvement in ECEC in China. On the one hand, men were actively welcomed to ECEC for the purposes of teaching children (especially boys) masculine ways of being socialized as citizens. On the other hand, there were doubts about gender identity and even personal integrity of men who had chosen employment as an ECT. Working in ECEC is also deemed to be inadequate for men because of the feminised nature of the job and its low socio-economic status. Further research is necessary to address how these paradoxes might contribute to transforming gender structures in ECEC centres in China. 


\section{Conclusion}

This study demonstrated that Chinese male and female ECTs' professional subjectivities of ECEC are closely aligned with traditional gender stereotypes in China, albeit some emerging evidence indicate that they would occasionally challenge essentialist gender views. Further, in a global context of empowering women and girls (United Nations 2015), the Chinese appreciation of masculinity in ECEC may disadvantage women and girls and will hardly contribute to achieving gender equality and diversity in Chinese society. It would thus be important to follow up moves to encourage and support men working in ECEC by some provincial governments in China (Jiangsu Education 2014; MENTEACH 2015). It is expected that these policies may have the potential to facilitate changes in public understanding of ECEC and its gendered characteristics as discussed in this article, rather than reproducing traditional gender stereotypes of men and women.

It is acknowledged that findings of this research have relied on male and female ECTs' selfreported subjectivities and therefore, may not represent a comprehensive picture about gender in action in ECEC in China. Observations on the actual daily practices in ECEC settings in China could offer alternative insights, including perspectives from other key stakeholders such as children and their parents. It was also noted in this research that there were some similarities between China and Western societies in terms of some gendered constructions of ECEC. Comparative studies on exploring men's participation in ECEC between China and other cultures could provide new possibilities for improving children's learning and development in the future. 


\section{References}

Blaise, M. 2005. Playing It Straight: Uncovering Gender Discourses in the Early Childhood Classroom. New York: Routledge.

Brandes, H., M. Andrä, W. Röseler, and P. S. Andrich. 2015. "Does Gender Make A

Difference? Results from the German 'Tandem Study' on the Pedagogical Activity of Female and Male ECE Workers." European Early Childhood Education Research Journal, 23:3, 315-327.

Brody, D. 2014. Men Who Teach Young Children: An International Perspective. London: Institute of Education Press.

Brownhill, S. 2015. "The 'Brave' Man in the Early Years (0-8): Defining the 'Role Model'." European Early Childhood Education Research Journal, 23:3, 370379.

Cao, K., and X. Wu. 2016. "Current Situations, Reasons, and Corresponding Solutions on the Lack of Prospective Male Kindergarten Teachers." [In Chinese.] Educational Management.

Chen, Y. 2015. "The Causes and Countermeasures of Male Teachers' Shortage in Kindergartens - Based on Zaozhang District." [In Chinese.] Journal of Zaozhuang Universirty, 32:4, 121-124.

Craig, D. A. 2004. "Males Studying and Working in Early Childhood Education: Advantages, Barriers, Challenges and Examples of Successful Strategies to Recruit, Retain and Support Men." Engaging Fathers Project, Family Action Centre, University of Newcastle, NSW.

Connell, R., and R. Pearse. 2015. Gender in World Perspective. 3rd ed. Cambridge: 
Polity Press.

Fereday, J., and E. Muir-Cochrane. 2006. "Demonstrating Rigor Using Thematic Analysis: A Hybrid Approach of Inductive and Deductive Coding and Theme Development.” International Journal of Qualitative Methods, 5(1), Article 7.

Han, X. 2016. “Analyses on the Lack of Male Kindergarten Teachers in China.” [In Chinese.] Technology and Economic Guide, 19, 140.

Hou, X., and X. Zhang. 2009. "Attributes to and Solutions on the Lack of Male Kindergarten Teachers in China." [In Chinese.] Early Childhood Education (Educational Sciences), No.10, General No. 454, 32-35.

Hu, B. Y. 2015. "Comparing Cultural Differences in Two Quality Measures in Chinese Kindergartens: The Early Childhood Environment Rating Scale-Revised and the Kindergarten Quality Rating System.” Compare: A Journal of Comparative and International Education, 45:1, 94-117.

Huang, L. 2013. "Interacting with Low Self-esteem: Case Study on Male Kindergarten Teachers' Social Relationships.” [In Chinese.] Journal of HuBei TV University, 33:2, 150. Huo, L., S.B. Neuman, and A. Nanakida. (eds). 2015. Early Childhood Education in Three Cultures: China, Japan and the United States. Heidelberg: Springer.

James, N., and H. Busher. 2006. "Credibility, Authenticity and Voice: Dilemmas in Online Interviewing." Qualitative Research, 6(3), 403-320.

Jiangsu Education. 2014. “The Training of Fee-free Pre-service Male Kindergarten Teachers Achieved Satisfactory Results." [In Chinese.] Accessed January 5, 2017. http://www.ec.js.edu.cn/art/2014/5/26/art_4336_149253.html 
Jing, S. 2016. "The Lack Current Situation and Influence of Male Kindergarten Teachers in China: From the Perspective of Infant's Sex Degree Development." [In Chinese.] Modern Education Science, 11, 117-122.

King, J. R. 2004. "The (Im)possibility of Gay Teachers for Young Children.” Theory into Practice, 43:2, 122-127.

Koch, B., and S. Farquhar. 2015. "Breaking Through the Glass Doors: Men Working in Early Childhood Education and Care with Particular Reference to Research and Experience in Austria and New Zealand." European Early Childhood Education Research Journal, 23:3, 380-391.

Li, J. 2015. "Discussions on the Impacts of Male Preschool Teachers on Child Development." [In Chinese.] Studies of Preschool Education, Good Parents.

Li, M., F. Deng, and L. Liu. 2015. "How to Advance the Initial Training System For Chinese Kindergarten Teachers in a New Era." In Early childhood education in Three Cultures: China, Japan and the United States, edited by L. Huo, S. B. Neuman, and A. Nanakida, 127-141. Heidelberg: Springer.

Li, X., Y. Liu, T. Duan, and J. Li. 2016. "Why Do They Choose Preschool Education: A Qualitative Study on Boys' Choice of Major and Professional Identity.” [In Chinese.] Early Childhood Education (Educational Sciences), No.1/2, General No. 681/682, 57-62. Li, T., and Q. Lu. 2013. "The Survival Situation and Rational Thinking of Male School Teachers." [In Chinese.] Education and Teaching Research, 27:3, 122-125. Little, H. 2015. "Promoting Risk-taking and Physically Challenging Play in Australian Early Childhood Settings in a Changing Regulatory Environment." Journal of Early Childhood 
Research, Online first. Doi:10.1177/1476718X15579743

Liu, W. 2013. “A Narrative Case Study on Male Kindergarten Teachers' Professional Development." [In Chinese.] Journal of Changchun Education Institute, 29:20, 68-69. Lewis-Beck, M. S., A. Bryman, and T. Futing. 2004. The Sage Encyclopedia of Social Science Research Methods (three volumes). Thousand Oaks, Calif.: Sage. MENTEACH. 2015. "Data About Men Teachers: Current Population Survey.”US Bureau of Labor Statistics. Accessed January 16,2016 . http://www.menteach.org/resources/data_about_men_teachers

MENTEACH. 2015. "China to Offer Free Teacher Training to Men.” Accessed January 18, 2016. http://www.menteach.org/node/2641

Ministry of Education of the People's Republic of China. 2014. "Number of Female Educational Personnel and Full-time Teachers of Schools by Type and Level.”[In Chinese.] Accessed January 16, 2016. http://old.moe.gov.cn/ publicfiles/business/htmlfiles/moe/s8493/201412/181717.html

National Bureau of Statistics of China. 2015. "Yearly Statistics 2014."

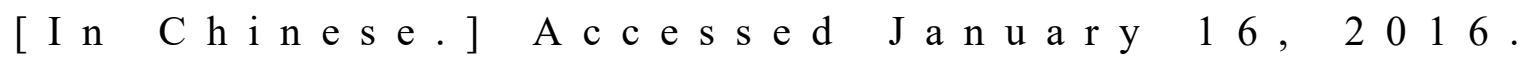
http://data.stats.gov.cn/easyquery.htm? $\mathrm{cn}=\mathrm{C} 01$

OECD. 2014. Education at a Glance 2014: OECD Indicators. OECD Publishing. Peeters, J. 2007. "Including Men in Early Childhood Education: Insights from the European Experience." NZ Research in Early Childhood Education, 10, 15-24. Peeters, J. 2013. “Towards a Gender Neutral Interpretation of Professionalism in Early Childhood Education and Care." Revista Española de Educación Comparada, 21: 
119-144.

Peeters, J., T. Rohrmann, and K. Emilsen. 2015. "Gender Balance in ECEC: Why Is There So Little Progress." European Early Childhood Education Research Journal, $23: 3,302-314$

Rentzou, K., and K. Ziganitidou. 2009. “Greek Male Early Childhood Educators: Self and Societal Perceptions Towards Their Chosen Profession." Early Years: An International Journal of Research and Development, 29:3, 271-279.

Robb, M. 2001. "Men Working in Childcare.” In Children in Society: Contemporary Theory, Policy and Practice, edited by P. Foley, J. Roche, and S. Tucker. Hampshire: Palgrave \& Open University.

Roberts-Holmes, G., and S. Brownhill. 2012. "Where Are the Men? A Critical Discussion of Male Absence in the Early Years.” In Professionalization, Leadership and Management in the Early Years, edited by L. Miller, and C. Cable. London: SAGE.

Rohrmann, T., and D. Brody. 2015. "Questioning Methodologies in Research on Men in ECEC." European Early Childhood Education Research Journal, 23:3, 405-416. Rohrmann, T., and K. Emilsen. 2015. “Editorial." European Early Childhood Education Research Journal, 23:3, 295-301.

Sak, R., i. T. Ş. Sak, and i. Yerlikaya. 2015. "Behavior Management Strategies: Beliefs and Practices of Male and Female Early Childhood Teachers." European Early Childhood Education Research Journal, 23:3, 328-339.

Sandseter, E. B. H. 2012. "Restrictive Safety or Unsafe Freedom? Norwegian ECEC 
Practitioners' Perceptions and Practices Concerning Children's Risky Play.” Child Care in Practice, 18(1), 83-101.

Shen, H. 2016. "A Study of Strategy for the Management of Male Kindergarten Teachers: Taking Z Kindergarten in Shanghai as An Example.” [In Chinese.] Early Childhood Education (Educational Science), No. 7/8, General No. 699/700, 41-44.

Smith, J. 2004. "Male Primary Teachers: Disadvantaged or Advantaged?." Paper presented to the Australian Association for Research in Education Conference, December 2004, Melbourne.

Twinn, S. 1997. “An Exploratory Study Examining the Influence of Translation on the Validity and Reliability of Qualitative Data in Nursing Research." Journal of Advanced Nursing, 26, 418-423.

United Nations. 2015. "Sustainable Development Goals.” Accessed December 30, 2016. https://sustainabledevelopment.un.org/?menu=1300

Warin, J. (2014) “The Status of Care: Linking gender and 'educare'." Journal of Gender Studies, 23:1, 93-106.

Williams, C.L. 1989. Gender Differences at Work: Women and Men in Nontraditional Occupations. London: University of California Press, Berkeley.

Xu, Y. 2012. "The Professional Self-identities of Male Early Years Teachers." MA diss., London, King's College.

Xu. P. 2016. "Discussions on the Advantages of Male Preschool Teachers on Child Development." [In Chinese.] New West, 21, 168-169.

Yang, J. 2014. "Analysis on Issues and Measures of Male Kindergarten Teachers' Gender 
Identities." [In Chinese.] Heilongjiang Education - Theory and Practice, 5, 13-14.

Zhao, Y. 2015. “Analysis of Male Kindergarten Teachers' Current Career Situations and Developments.” [In Chinese.] New Curriculum Studies, 01, 49-50.

Zhao, Y. 2016. "Literature Review on the Study of Male Preschool Teacher in Recent 10 Years - On the Basis of CNKI (2006-2016)." [In Chinese.] Journal of Shaanxi Xueqian Normal University, 32:10, 146-149.

Zheng, G. 2013. "Analysis on Reasons for the Hidden Loss of Male Kindergarten Teachers." [In Chinese.] Journal of Xinxiang University (Social Science Edition), 27:3, 128-130. 